\title{
Détermination de l'âge des jeunes migrants: Position de la Société Suisse de Pédiatrie
}

\begin{abstract}
Dr. méd. Sarah Depallens ${ }^{a}$, Dr. méd. Fabienne Jäger ${ }^{b}$, Dr. méd. Nicole Pellaud
Cette position a été validée par les experts de pédiatrie du développement: Prof. Dr. méd Oskar Jennid, Prof. Dr. méd Valérie Schwitzgebele, PD Dr. méd. Georg Eich', le comité de la société suisse de pédiatrie et son groupe santé migrant

a Service de pédiatrie CHUV Lausanne et groupe migrants SSP; ${ }^{b}$ Service de pédiatrie Hôpital du Jura et groupe migrants SSP; ${ }^{c}$ Présidente SSP, Service médical scolaire et psychopédagogique Sion; d Pédiatrie du développement, Kinderspital Zürich; e Endocrinologie pédiatrique, Hôpital des enfants HUG, Genève; ${ }^{\dagger}$ Radiologie pédiatrique, Kantonsspital Aarau
\end{abstract}

pour faciliter la lecture, la forme masculine est utilisée pour représenter les deux genres

\section{$\because$ ssp sgp}

Responsabilité rédactionnelle:

Société Suisse de Pédiatrie

La Société suisse de pédiatrie (SSP) souhaite informer les médecins ainsi que les instances fédérales et cantonales compétentes de la problématique qui se pose à propos de la détermination de l'âge des jeunes migrants*. Elle publie une position élaborée par des pédiatres spécialisées en santé des jeunes migrants et en pédiatrie sociale. En souhaitant que cette position puisse promouvoir en Suisse une prise en charge des jeunes migrants respectueuse des droits de l'enfant et des droits de l'homme.

Chaque année, la Suisse accueille des requérants d'asile. Ils étaient 27'207 en 2016 dont 7,3\% considérés comme des mineurs non accompagnés [1].

Parallèlement à d'autres critères, la procédure d'asile tient compte de l'âge du jeune requérant, les adolescents de plus de 18 ans n'ayant pas droit à l'encadrement réservé d'office aux mineurs (foyer avec éducateurs, protection par un tuteur, accès à l'école,...). Cette différence de traitement a un impact important pour le futur du jeune. Par exemple, l'accès à une formation étant un des facteurs protecteurs les plus important à l'adolescence, un mineur estimé comme majeur à son arrivée en Suisse sera dès son départ à risque de désinsertion et donc en danger dans son développement.

En Suisse, afin de déterminer l'âge des jeunes qui ne possèdent pas de documents valables attestant leur date de naissance, les institutions gouvernementales font parfois appel aux médecins.

Sur le plan de la déontologie médicale, tout examen n'est réalisé qu'au respect des conditions suivantes 1) indication médicale pour l'amélioration de la santé 2) consentement éclairé de la personne 3) méthode validée et sans danger [2,3].

Dans le cadre d'un mandat non thérapeutique, la personne doit être clairement informée et le médecin ne peut pas avoir recours à des pratiques diagnostiques discutables [3].

Or, pour ces déterminations, comme cela a déjà été démontré et publié à diverses reprises [4, 5], que cela soit

Aujourd'hui, aucune méthode scientifique ne permet d'établir précisément l'âge d'un jeune qui se situerait entre 15 et 20 ans.

pour l'âge osseux [6, 7, 8, 11], l'examen physique [8] ou dentaire $[9,10,12]$, ces outils sont trop approximatifs et présentent des écarts-types larges, ils se fondent sur des tables de références souvent non adaptées car ne tenant compte ni de l'origine ethnique $[11,12]$ ou socioéconomique du jeune, ni d'éventuelles pathologies endocriniennes [8] pouvant influencer les résultats. S'y ajoute une irradiation inutile. 
Aujourd'hui, aucune méthode scientifique ne permet d'établir précisément l'âge d'un jeune qui se situerait entre 15 et 20 ans afin de définir avec certitude s'il est majeur ou mineur: en effet des valeurs d'adultes peuvent être trouvées chez un jeune mineur conduisant à une surévaluation de son âge.

Une évaluation globale par des professionnels spécialisés dans le développement de l'adolescent reste pertinente afin d'évaluer le développement du jeune, son

\section{La Société suisse de pédiatrie recommande à ses membres et à tout médecin sollicité de ne pas participer à la détermination de l'âge des jeunes requérants d'asile.}

statut psycho-cognitif, et pouvoir si nécessaire l'orienter vers des structures qui puissent tenir compte de ses vulnérabilités [13].

Dans un contexte où la détermination de l'âge ne répond pas aux critères déontologiques et comporte un risque d'erreur avec des conséquences légales et sociales majeures sur la vie de ces jeunes, la Société suisse de pédiatrie, comme l'ont déjà fait plusieurs sociétés et académies internationales de pédiatrie $[14,15,16]$ recommande à ses membres et à tout médecin sollicité de ne pas participer à la détermination de l'âge des jeunes requérants d'asile et de prendre position dans ce sens auprès des institutions cantonales en charge de la migration.

\section{Références}

$1 \mathrm{https} / / /$ asile.ch/statistiques/suisse/

2 Charte du pédiatre, SSP 2017

3 FMH Standesordnung Art 6, 8, http://www.fmh.ch/files/pdf18/ Standesordnung_August_2016_D.pdf

4 Aynsley-Green A, Cole TJ, Crawley H, Lessof N, Boag LR, Wallace RM. Medical, statistical, ethical and human rights considerations in the assessment of age in children and young people subject to immigration control. Br Med Bull. 2012;102:17-42.

5 Hjern A, Brendler-Lindqvist M, Norredam M. Age assessment of young asylum seekers. Acta paediatr. (Oslo, Norway: 1992). 2012;101(1):4-7.5

6 Cole TJ. The evidential value of developmental age imaging for assessing age of majority. Ann Hum Biol. 2015;42(4):379-88.

7 Thodberg Hans Henrik, van Rijn Rick R, Jenni Oskar G, Martin David D. Automated determination of bone age from hand X-rays at the end of puberty and its applicability for age estimation. Int J Legal Med. DOI 10.1007/s00414-016-1471-8

8 Schwitzgebel V. Georg F. L'âge osseux ne permet pas de déterminer l'âge des jeunes requérants d'asile, Paediatrica Vol. 27 No. 3, 292016

9 Knell B. 2012. Zahnärztliche Altersdiagnostik zur Frage nach dem 18. Altersjahr. Kriminalistik-Schweiz. 2/12, 122-127, 2012.

10 Reutimann Felix. Zahnärztliche Altersdiagnostik: Untersuchung zur radiologischen Sichtbarkeit des Parodontalspaltes der ersten Molaren im Unterkiefer bei 14- bis 22-Jährigen. 2015, University of Zurich, Faculty of Medicine http://www.zora.uzh.ch/123990/1/ Dissertation_Reutimann_12_12_2015.pdf

11 Ontell FK, Ivanovic M, Ablin DS, Barlow TW. Bone age in children of diverse ethnicity, http://www.ajronline.org/doi/abs/10.2214/ ajr.167.6.8956565

12 Olze A, Schmeling A, Taniguchi M, Maeda H, van Niekerk P, Wernecke KD, Geserick G. Forensic age estimation in living subjects: the ethnic factor in wisdom tooth mineralization. Int J Legal Med. 2004;118(3):170-3. Epub 2004 Feb 6

13 Messelken D, Crouse J. When childhood ends: estimating the age of young people BMJ. 2015;351:h6699 doi: 10.1136/bmj.h6699

14 Royal College of Paediatrics and Child Health (2009) Policy: College statement on the role of paediatricians in the age assessment of unaccompanied young people seeking asylum, http://www. rcpch. ac.uk/Policy

15 On behalf of the Advocacy and Ethics Group of the European Academy of Paediatrics, PJJ Sauer, A Nicholson, D Neubauer. Age determination in asylum seekers: physicians should not be implicated. Eur J Pediatr. 2016;175(3):299-303.

16 International Society for Social Paediatrics Position Statement on Migrant Child Health 2017. 